\title{
Evaluation of a.c. conductivity of rubber ferrite composites from dielectric measurements
}

\author{
S SINDHU, M R ANANTHARAMAN*, BINDU P THAMPI, K A MALINI and \\ PHILIP KURIAN ${ }^{\dagger}$ \\ Department of Physics, 'Department of Polymer Science and Rubber Technology, Cochin University of Science \\ and Technology, Cochin 682 022, India
}

MS received 26 November 2001; revised 19 April 2002

\begin{abstract}
The effect of frequency, composition and temperature on the a.c. electrical conductivity were studied for the ceramic, $\mathrm{Ni}_{1-x} \mathrm{Zn}_{x} \mathrm{Fe}_{2} \mathrm{O}_{4}$, as well as the filler $\left(\mathrm{Ni}_{1-x} \mathrm{Zn}_{x} \mathrm{Fe}_{2} \mathrm{O}_{4}\right)$ incorporated rubber ferrite composites (RFCs). $\mathrm{Ni}_{1-x} \mathrm{Zn}_{x} \mathrm{Fe}_{2} \mathrm{O}_{4}$ (where $x$ varies from 0 to 1 in steps of $0 \cdot 2$ ) were prepared by usual ceramic techniques. They were then incorporated into a butyl rubber matrix according to a specific recipe. The a.c. electrical conductivity $\left(\sigma_{\text {a.c. }}\right)$ calculations were carried out by using the data available from dielectric measurements and by employing a simple relationship. The a.c. conductivity values were found to be of the order of $10^{-3} \mathrm{~S} / \mathrm{m}$. Analysis of the results shows that $\sigma_{\text {a.c. }}$ increases with increase of frequency and the change is same for both ceramic $\mathrm{Ni}_{1-x} \mathrm{Zn}_{x} \mathrm{Fe}_{2} \mathrm{O}_{4}$ and RFCs. $\sigma_{\text {a.c. }}$ increases initially with the increase of zinc content and then decreases with increase of zinc. Same behaviour is observed for RFCs too. The dependence of $\sigma_{\text {a.c. }}$ on the volume fraction of the magnetic filler was also studied and it was found that the a.c. conductivity of RFCs increases with increase of volume fraction of the magnetic filler. Temperature dependence of conductivity was studied for both ceramic and rubber ferrite composites. Conductivity shows a linear dependence with temperature in the case of ceramic samples.
\end{abstract}

Keywords. Rubber ferrite composites; a.c. electrical conductivity; mixed ferrites; electrical properties; magnetic materials; polymer magnets.

\section{Introduction}

Ferrites constitute an important group of magnetic materials with a wide range of applications due to their magnetic properties and low dielectric loss (Elhiti 1994; Smit and Wijn 1959). Mixed ferrites like nickel zinc ferrites have low eddy current loss and high resistivity. Hence, they are more important commercially (Kulikowski 1984). The electrical properties are important for ferrites and composites containing mixed ferrites, not only from the application point of view but also from the fundamental point of view (Yootarou and Minuru 1973; Pal et al 1994; Ahmed and Elhiti 1995; Elhiti et al 1995; Ahmed et al 1996; Andrej and Miha 1999; Shaikh et al 1999). Evaluation of magnetic and dielectric properties of the composites and correlation of results may help in tailoring composites for various applications. Evaluation of a.c. electrical conductivity reveals a wealth of information as regards the usefulness of these materials for various applications. Moreover the study of a.c. electrical conductivity sheds light on the behaviour of charge carriers under an a.c. field, their mobility and the mechanism

*Author for correspondence of conduction (Koops 1951; Brockman and White 1971; Jankowski 1988). The conductivity studies on nickel zinc ferrites were carried out by various researchers (Samakhvalov and Rustamov 1964; Chandra Prakash Rana and Baijal 1985; Elhiti 1996; Pal et al 1996; Abdeen 1998). However, a survey of literature reveals that the conductivity studies on ferrite incorporated rubber composites are rather scarce or seldom reported.

Rubber ferrite composites (RFCs) are important in that they are composite materials suitable for devices where flexibility is an important parameter. Moreover, these composites can be moulded into complex shapes. They are also excellent materials as microwave absorbers (Mirtaheri et al 1989; Kim et al 1991; Stoyan et al 1994; Sung Soo et al 1994; Yoshiyuki et al 1998). In principle, they are essentially very good dielectrics with appropriate magnetic properties. RFCs are essentially dielectric materials and a.c. conductivity plays an important role. So study of these materials, particularly in an a.c. field assumes significance.

Incorporation of mixed ferrites viz. nickel zinc ferrites, in various matrices lead to rubber ferrite composites (Anantharaman et al 1998). The composites should have the required magnetic, mechanical and dielectric properties. The incorporation of these ferrites is carried out according to a specific recipe for mixing. It is then 
customary to use pre-characterized ceramic fillers, which is impregnated into the matrix to make the RFCs. The present study includes evaluation of a.c. electrical conductivity for both ceramic $\mathrm{Ni}_{1-x} \mathrm{Zn}_{x} \mathrm{Fe}_{2} \mathrm{O}_{4}$ as well as the filler incorporated composites. It is also to be noted that the evaluation of a.c. conductivity for both ceramic and RFCs have been carried out using the data made available from the dielectric measurements conducted on these samples, in the frequency range $100 \mathrm{~Hz}-10 \mathrm{MHz}$ (Goswami 1996; Anantharaman et al 1999). This method was adopted as $\tan \delta$ (dielectric loss) values were also available during the measurement of dielectric permittivity by using impedance analyser and a dielectric cell. Thus by this experimental set-up it is possible to evaluate the a.c. conductivity values, loss factor and dielectric values in a single shot and also it is possible to carry out the frequency and temperature variation studies on these samples.

\section{Experimental}

\subsection{Sample preparation and structural characterization}

Mixed ferrites containing nickel and zinc belonging to the series, $\mathrm{Ni}_{1-x} \mathrm{Zn}_{x} \mathrm{Fe}_{2} \mathrm{O}_{4}$ for various $x$ ( $x=0$ to 1 in steps of 0.2 ) were prepared by employing ceramic techniques. For this, precursors viz. ferrous oxalate dihydrate, nickel oxide and zinc oxide in appropriate weight ratio were mixed thoroughly in an agate mortar to produce a homogeneous mixture of fine particles. Repeated sintering at $500^{\circ} \mathrm{C}$ and mixing of this powder were continued till single phasic spinel NZF were obtained. This pre-sintered powder was then finally sintered at $1000 \pm 15^{\circ} \mathrm{C}$ for several hours. These powder samples thus prepared by the mixed oxide route were dispersed in a butyl rubber matrix for various loadings of the magnetic filler such as $20 \mathrm{phr}, 40$ phr, $80 \mathrm{phr}, 120 \mathrm{phr}$ ( $\mathrm{phr}$ - parts per hundred rubber by weight) according to a specific recipe. The recipe and conditions for mixing are cited elsewhere (Anantharaman et al 1998).

Identification of the phase was carried out in the ceramic ferrites by using X-ray powder diffractometer(XRD). The X-ray diffractograms of these powder samples were recorded on a Philips (PW1130) X-ray diffractometer using $\mathrm{CuK}_{\alpha}$ radiation $(\lambda=1.5418 \AA)$.

\subsection{Magnetic and dielectric measurements}

Magnetic characterization of both ceramic $\mathrm{Ni}_{1-x} \mathrm{Zn}_{x} \mathrm{Fe}_{2} \mathrm{O}_{4}$ and rubber ferrite composites were carried out by using vibrating sample magnetometer (VSM) (model: EG \& G PARC 4500). Parameters like saturation magnetization $\left(M_{\mathrm{S}}\right)$, retentivity $\left(M_{\mathrm{r}}\right)$, coercivity $\left(H_{\mathrm{c}}\right)$ and energy loss were obtained from the hysteresis loop obtained from VSM measurements.
The dielectric studies of both ceramic and rubber ferrite composites were carried out by using a dielectric cell and an impedance analyser (model: HP 4192A). The details of these measurements are cited elsewhere (Anantharaman et al 1999). Disc shaped samples were used to find out the dielectric constant. The capacitance and dielectric loss in the frequency range $100 \mathrm{kHz}-10 \mathrm{MHz}$ were found out. Dielectric constant or relative permittivity were calculated by using the formula

$$
\varepsilon_{\mathrm{r}}=\frac{C \times d}{\varepsilon_{0} A}
$$

where $d$ is the thickness of the sample, $C$ the capacitance and $A$ the area of cross section of the sample. $\varepsilon_{\mathrm{r}}$ is the relative permittivity of the material which is a dimensionless quantity. From these measurements, $\varepsilon_{\mathrm{r}}$ and $\tan \delta$ (dielectric loss factor), for both ceramic and RFCs were made available for the evaluation of a.c. conductivity on these samples.

\subsection{Principle and theory (Evaluation of $\sigma_{\text {a.c. }}$ from dielectric measurements)}

A capacitor when charged under an a.c. voltage will have some loss current due to ohmic resistance or impedance by heat absorption. If $Q$ be the charge in coulombs due to a potential difference of $V$ volts between two plates of a condenser of area, $A$, and interplate distance, $d$, then a.c. conductivity $\left(\sigma_{\text {a.c. }}\right)$ due to a.c. voltage $v\left(v_{0} e^{j \omega t}\right)$ is given by the relation

$$
\sigma_{\text {a.c. }}=\frac{J}{E},
$$

$J$ is the current density and $E$ the electric field strength vector.

But the electric field vector, $E=D / \varepsilon$. $D$ is the displacement vector of the dipole charges. $\varepsilon$ is the complex permittivity of the material. For a parallel plate capacitor the electric field intensity $(E)$ is the ratio of potential difference between the plates of the capacitor to the inter plate distance. i.e.

$$
E=\frac{V}{d}
$$

Since the current density $J=\mathrm{d} q / \mathrm{d} t$ but $q$ is given by

$$
\begin{aligned}
& \frac{Q}{A}=\frac{V \hat{\mathrm{A}}}{d}, \\
& \therefore J=\frac{\mathrm{d} q}{\mathrm{~d} t}=\frac{\mathrm{d}}{\mathrm{d} t}\left(\frac{V \varepsilon}{d}\right)=\frac{\varepsilon}{d} \frac{\mathrm{d} V}{\mathrm{~d} t},
\end{aligned}
$$

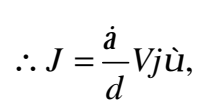

substituting for $E$ and $J$ in (2) 


$$
\sigma_{\text {a.c. }}=\frac{J}{E}=\varepsilon j \omega,
$$

since $\varepsilon$ being a complex quantity

$$
=\left(\varepsilon^{1}-j \varepsilon^{11}\right) j \omega=\varepsilon^{1} j \omega+\omega \varepsilon^{11},
$$

in order that a.c. conductivity may be a real quantity, the term containing $j$ has to be neglected hence

$$
\sigma_{\text {a.c. }}=\omega \varepsilon^{11} \text {. }
$$

In any dielectric material there will be some power loss because of the work done to overcome the frictional damping forces encountered by the dipoles during their rotation. If an a.c. field is considered, then in an ideal case the charging current $I_{\mathrm{C}}$ will be $90^{\circ}$ out of phase with the voltage. But in most of the capacitors due to the absorption of electrical energy some loss current, $I_{\mathrm{L}}$ will also be produced, which will be in phase with the voltage. Charging current, $I_{\mathrm{C}}$, and loss current, $I_{\mathrm{L}}$, will make angles $\delta$ and $\theta$, respectively with the total current, $I$, passing through the capacitor. The loss current is represented by $\sin \delta$ of the total current, I. Generally, $\sin \delta$ is called the loss factor but when $\delta$ is small then $\sin \delta=\delta=\tan \delta$. But the two components $\varepsilon^{1}$ and $\varepsilon^{11}$ of the complex dielectric constant, $\varepsilon$, will be frequency dependent and is given by

$$
\varepsilon^{1}(\omega)=D_{0} \cos \delta / E_{0}
$$
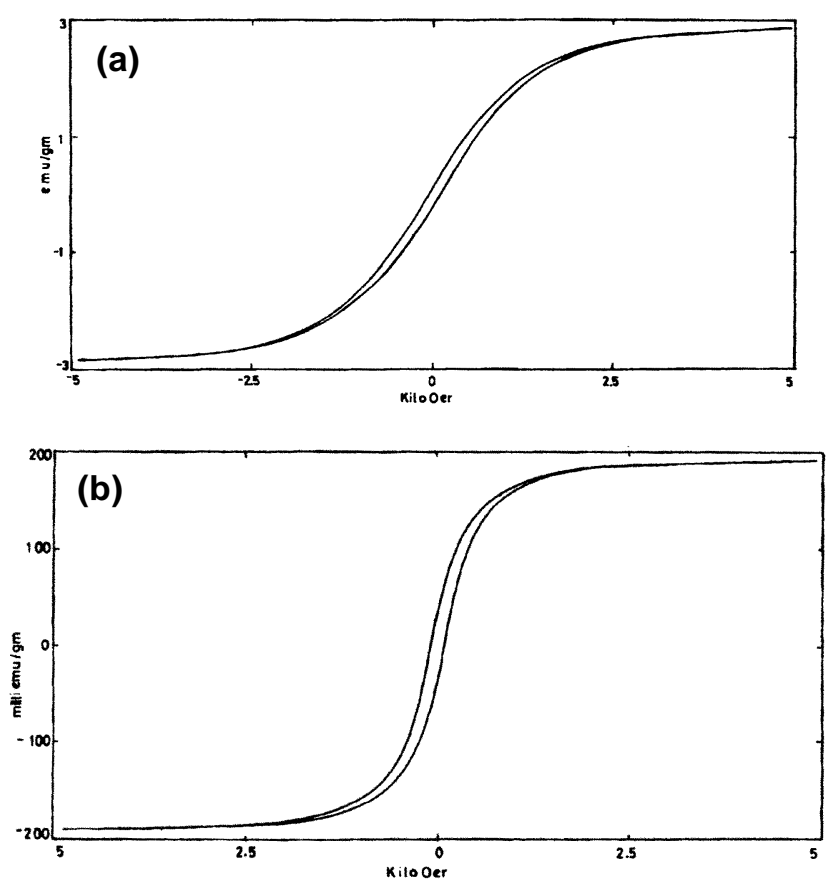

Figure 1. Representative hysteresis loops for (a) ceramic $\mathrm{Ni}_{0 \cdot 4} \mathrm{Zn}_{0 \cdot 6} \mathrm{Fe}_{2} \mathrm{O}_{4}$ and (b) RFC ( $\left.x=0,20 \mathrm{phr}\right)$.

$$
\varepsilon^{11}(\omega)=D_{0} \sin \delta / E_{0},
$$

since the displacement vector in a time varying field will not be in phase with $E$ and hence there will be a phase difference $\delta$ between them.

From (8) and (9), we have

$$
\tan \delta=\frac{\varepsilon^{11}(\omega)}{\varepsilon^{1}(\omega)} .
$$

Substituting the value of $\varepsilon^{11}(\omega)$ from (10) in (7) then we have

$$
\sigma_{\text {a.c. }}=\omega \tan \delta \varepsilon^{1}(\omega)
$$

where $\omega=2 \pi f$ and $\varepsilon^{1}=\varepsilon_{0} \varepsilon_{\mathrm{r}}$, here $\varepsilon_{\mathrm{r}}$ is the relative permittivity of the material and $\varepsilon_{0}$ the permittivity of free space. So

$$
\sigma_{\text {a.c. }}=2 \pi f \tan \delta \varepsilon_{0} \varepsilon_{\mathrm{r}} .
$$

This equation is used to calculate the a.c. conductivity using dielectric constant and $\tan \delta$ at a given frequency. It is to be noted that both $\tan \delta$ and $\varepsilon_{\mathrm{r}}$ were available from dielectric measurements.
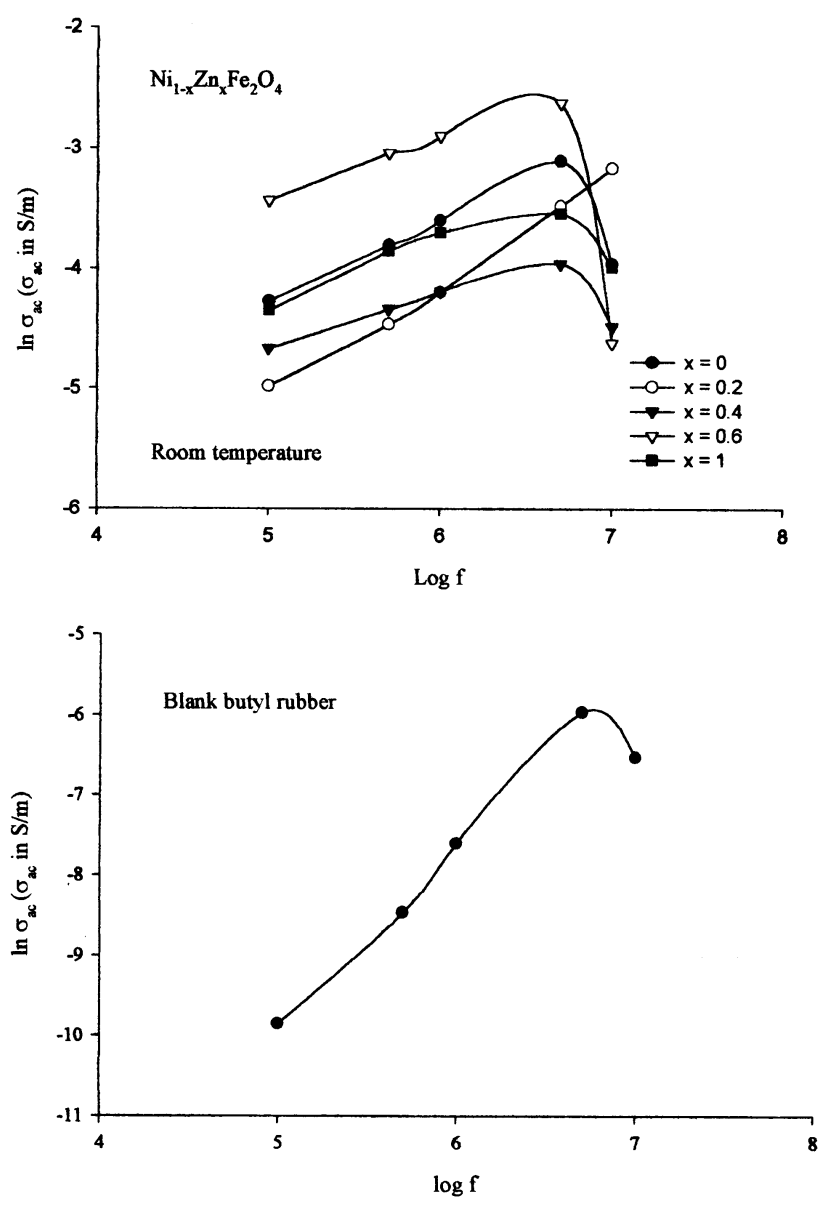

Figure 2. Frequency dependence of conductivity for $\mathrm{Ni}_{1-x} \mathrm{Zn}_{x} \mathrm{Fe}_{2} \mathrm{O}_{4}$ blank butyl rubber (room temperature). 


\section{Results and discussion}

Magnetic characterization of ceramic nickel zinc ferrites and the RFCs were carried out and analysed in detail and the results are cited elsewhere (Anantharaman et al 1998, 2001). Representative hysteresis loop for an RFC is shown in figure 1 . The detailed analysis of the electrical properties of these samples are discussed below.

\subsection{Frequency dependence}

The a.c. electrical conductivity of ceramic nickel zinc ferrites and rubber ferrite composites has been computed for different frequencies $(100 \mathrm{kHz}$ to $10 \mathrm{MHz})$ and also at different temperatures (varying from $303 \mathrm{~K}$ to $473 \mathrm{~K}$ ). It was observed that the a.c. electrical conductivity increases with increase of frequency initially and it shows a
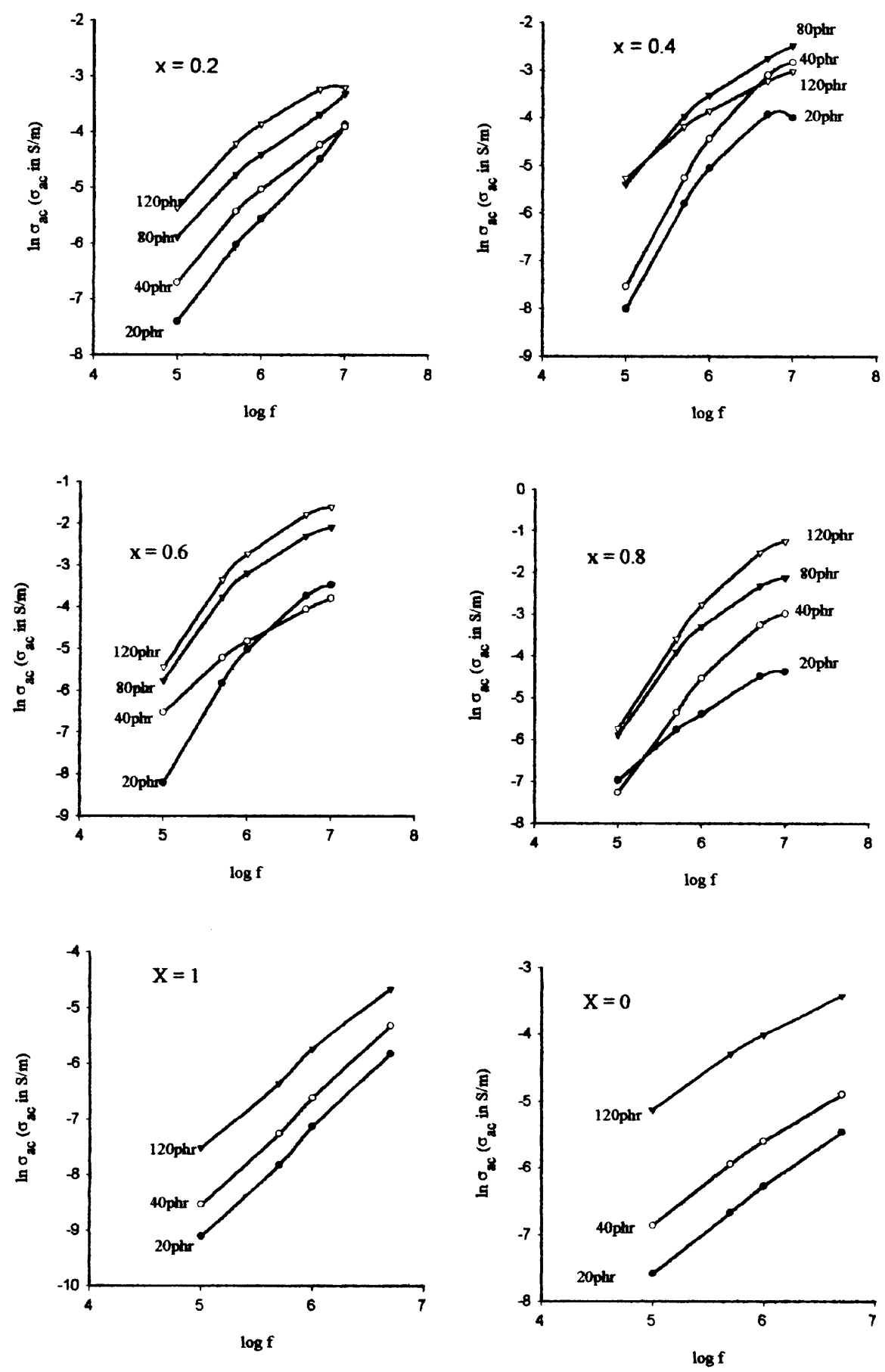

Figure 3. Variation of conductivity with frequency for rubber ferrite composites. 
small dip at higher frequencies that is above $5 \mathrm{MHz}$. This variation is same for samples with different concentrations of zinc ions. Frequency dependence of conductivity

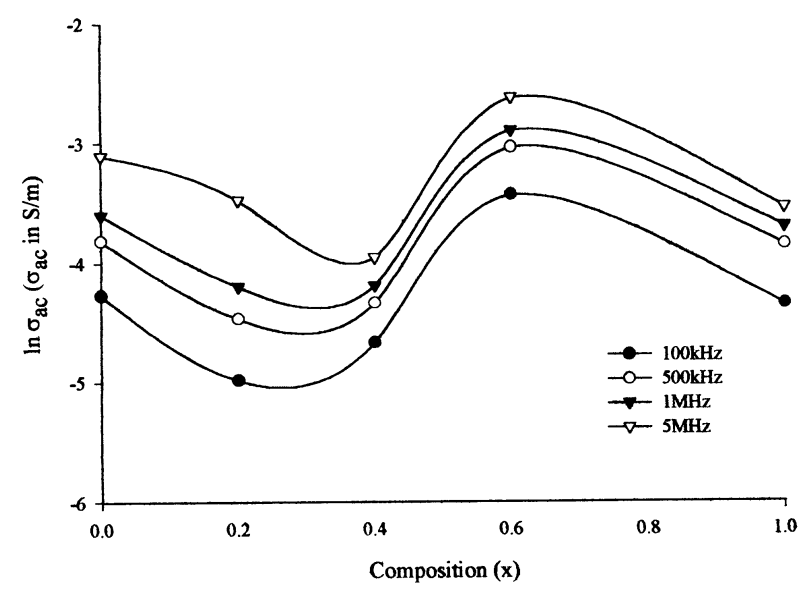

Figure 4. Variation of conductivity with composition for ceramic $\mathrm{Ni}_{1-x} \mathrm{Zn}_{x} \mathrm{Fe}_{2} \mathrm{O}_{4}$.
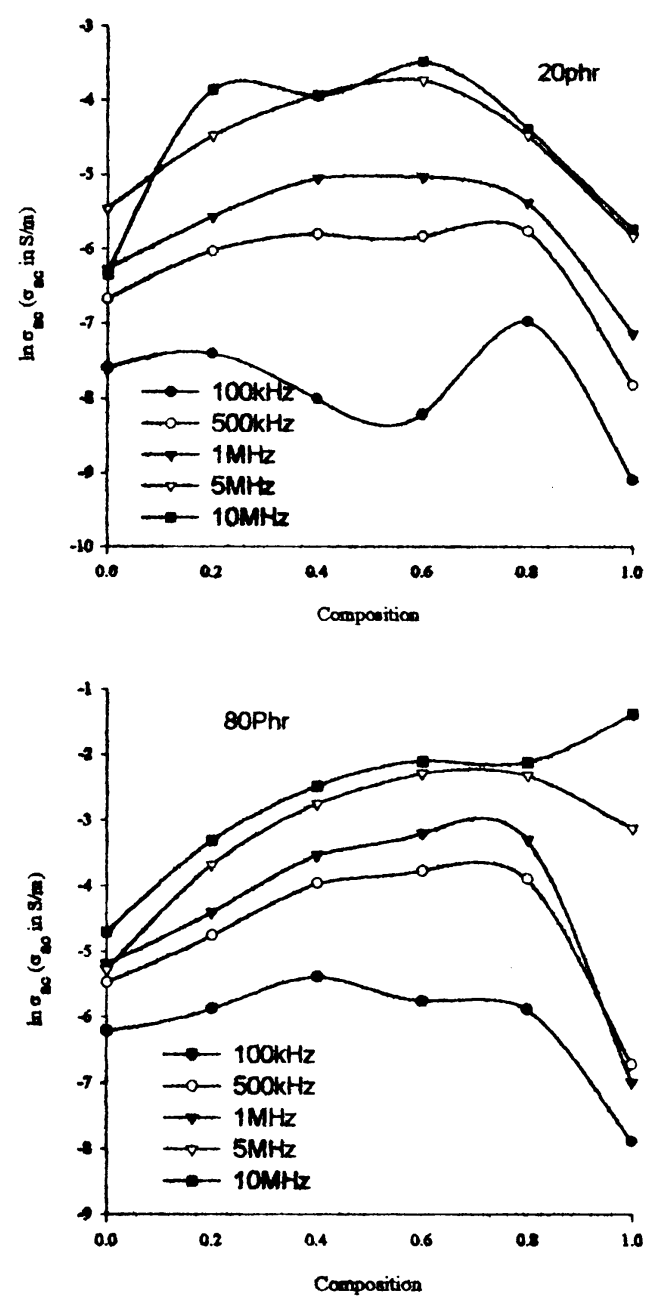

for blank butyl rubber was also noted. It also shows a small decrease after $5 \mathrm{MHz}$. Figure 2 shows the dependence of conductivity with frequency for both ceramic nickel zinc ferrites and blank butyl rubber. The hopping of electron between $\mathrm{Fe}^{2+}$ and $\mathrm{Fe}^{3+}$ ions on the octahedral sites is responsible for conduction in ferrites. Also hole hopping between $\mathrm{Ni}^{2+}$ and $\mathrm{Ni}^{3+}$ on $\mathrm{B}$ site will also contribute to electric conduction in ferrites. In this $\mathrm{Ni}_{1-x} \mathrm{Zn}_{x} \mathrm{Fe}_{2} \mathrm{O}_{4}$ system the hole conduction depends on the concentration of $\mathrm{Zn}$ ions on the $\mathrm{A}$ site to the $\mathrm{Ni}$ ion concentration on B site. The frequency dependence can be explained with the help of Maxwell-Wagner two-layer model or the heterogeneous model of the polycrystalline structure of ferrites (Koops 1951). According to this theory two layers formed dielectric structure. The first layer consists of ferrite grains of fairly well conducting (ferrous ions), which is separated by a thin layer of poorly conducting substances, which forms the grain boundary. These grain boundaries are more active at lower frequencies, hence the hopping frequency of electron between $\mathrm{Fe}^{3+}$ and $\mathrm{Fe}^{2+}$ ion is less at lower
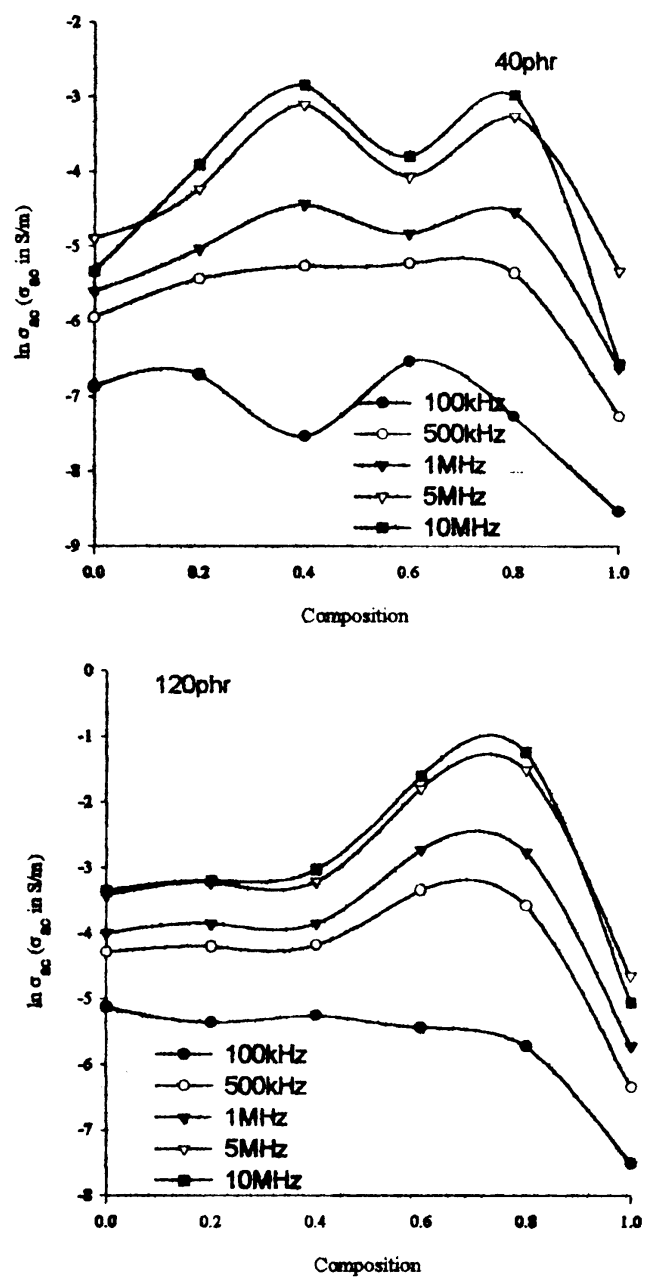

Figure 5. Variation of a.c. conductivity with composition for RFCs. 
frequencies. As the frequency of the applied field increases, the conductive grains become more active by promoting the hopping of electron between $\mathrm{Fe}^{2+}$ and $\mathrm{Fe}^{3+}$ ions, thereby increasing the hopping frequency. Thus we observe a gradual increase in conductivity with frequency. But at higher frequencies the frequency of hopping between the ions could not follow the applied field frequency and it lags behind it. This causes a dip in conductivity at higher frequencies. The same variation is observed for ferrite incorporated rubber samples too. Figure 3 shows the dependence of a.c. electrical conductivity with frequency for representative rubber ferrite composites. It was also noticed that this remains the same for samples with different volume fractions of the magnetic filler.

\subsection{Compositional dependence}

The variation of conductivity with composition (zinc content) for ceramic nickel zinc ferrites at room temperature is shown in figure 4. It was found that the a.c. electrical conductivity for $\mathrm{NiFe}_{2} \mathrm{O}_{4}$ (i.e. $x=0$ ) is greater than that for $\mathrm{Ni}_{0.8} \mathrm{Zn}_{0.2} \mathrm{Fe}_{2} \mathrm{O}_{4}$ and $\mathrm{Ni}_{0.6} \mathrm{Zn}_{0.4} \mathrm{Fe}_{2} \mathrm{O}_{4}$. In the $\mathrm{Ni}_{1-x} \mathrm{Zn}_{x} \mathrm{Fe}_{2} \mathrm{O}_{4}$ system when $\mathrm{x}=0$, maximum number of
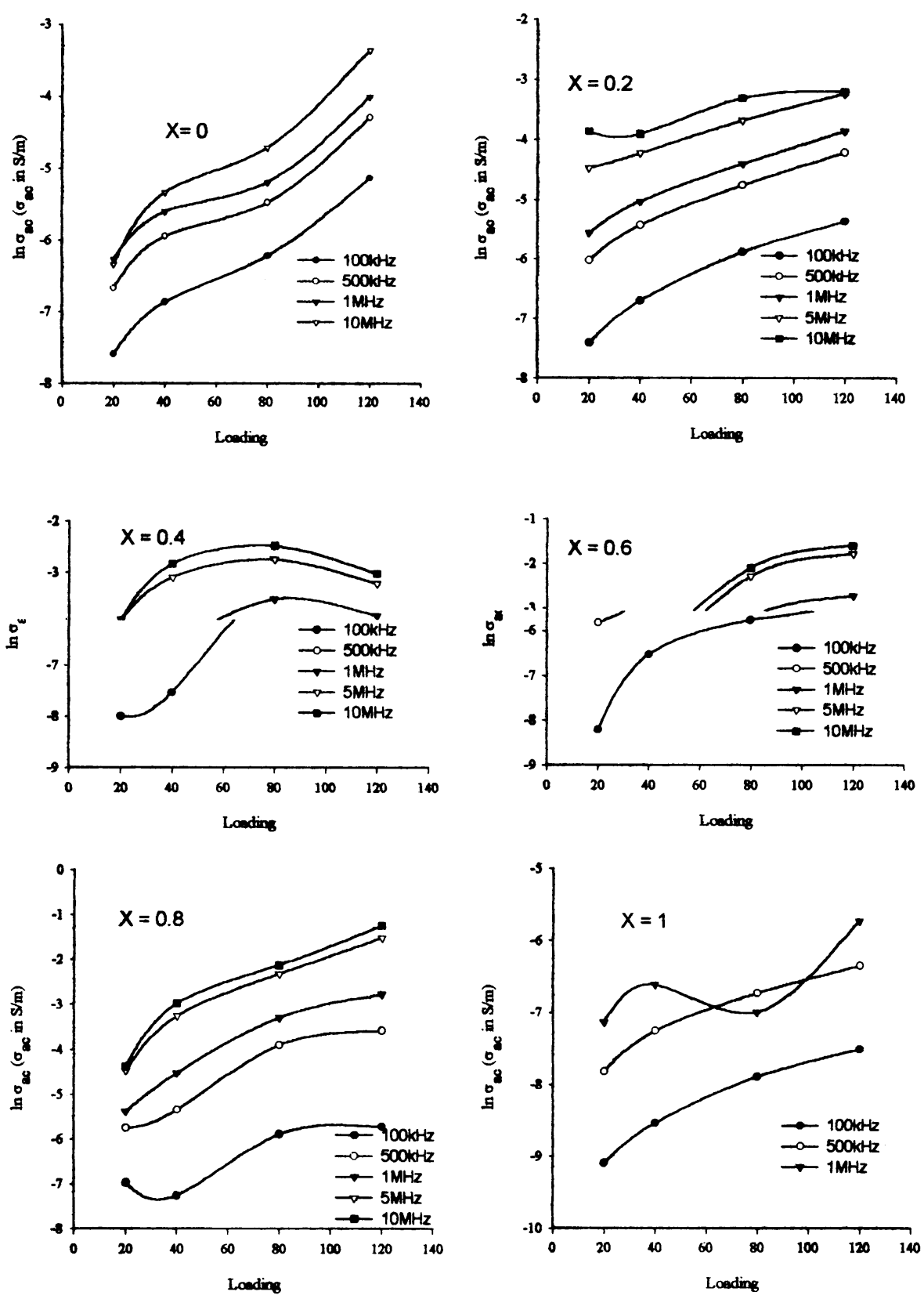

Figure 6. Variation of a.c. conductivity with loading for different compositions at different frequencies. 
$\mathrm{Ni}^{2+}$ and $\mathrm{Ni}^{3+}$ ions are available for hopping. Hence the main contribution of conductivity may be due to the hole hopping. As the zinc concentration $(x)$ increases, the number of $\mathrm{Ni}^{2+}$ and $\mathrm{Ni}^{3+}$ ions on $\mathrm{B}$ sites decreases. In the present work the small decrease in conductivity for composition $x=0.2$ and $x=0.4$ was observed, this may be due to the electron hole compensation in the B sites. Further increase of zinc concentration diminishes the hole hopping by decreasing the number of $\mathrm{Ni}^{2+}$ and $\mathrm{Ni}^{3+}$ ions on the $\mathrm{B}$ site. Thus electron hopping became predominant and it increases the conductivity. Thus we observe a maximum conductivity for $\mathrm{Ni}_{0.4} \mathrm{Zn}_{0.6} \mathrm{Fe}_{2} \mathrm{O}_{4}$, again it was observed that for $x=1$, i.e. for $\mathrm{ZnFe}_{2} \mathrm{O}_{4}$, the conductivity decreases. This decrease can be explained by considering the grain size. It was well known that the growth of grain and the formation of grain boundaries also influence the conductivity. In this present $\mathrm{NiZn}$ ferrite system it was observed that grain size first decreases with increase of $\mathrm{Zn}$ concentration and attained a minimum for $x=0 \cdot 6$. Thereafter it increased with $x$. Thus a maximum grain size of $96.44 \mathrm{~nm}$ was observed for $\mathrm{ZnFe}_{2} \mathrm{O}_{4}(x=1)$. The variation of grain sizes with zinc content have already been studied (Anantharaman et al 1999). As the grain size increases, the formation of oxygen rich layers on the surface of the grains and grain boundaries are possible. These non conducting layers increase the resistivity thereby decreasing the conductivity. This explains the decrease in conductivity at $x=1$. For rubber ferrite composites also the same variation is observed for all loadings. Variation pattern is shown in figure 5. The effect of matrix is minimal in this case.

\subsection{Loading dependence}

The addition of a conductive filler to a rubber or polymer is expected to impart reasonably good bulk conductivity.

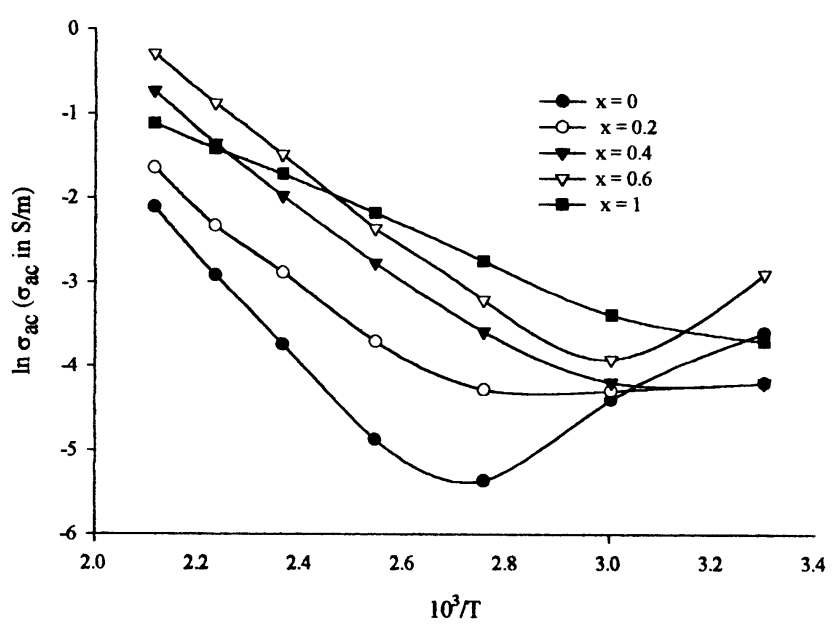

Figure 7. In $\sigma_{\text {a.c. }}$ vs $10^{3} / \mathrm{T}$ for ceramic $\mathrm{Ni}_{1-\gamma} \mathrm{Zn}_{x} \mathrm{Fe}_{2} \mathrm{O}_{4}(1 \mathrm{MHz})$.
In the case of butyl rubber containing $\mathrm{Ni}_{1-x} \mathrm{Zn}_{x} \mathrm{Fe}_{2} \mathrm{O}_{4}$ also increase in conductivity was observed with increase of the volume fraction of the filler. A maximum conductivity is observed for a maximum volume fraction of $120 \mathrm{phr}$. This is same for all compositions (i.e. for all $x$ values). Graphs showing the variation pattern is depicted in figure 6. Conductivity of the matrix at the lowest loading of the filler was affected by three parameters viz. the intrinsic conductivity of the filler, the shape of the filler and also the surface tension of the matrix and the filler (Terje 1986). It was expected that fibrous fillers will yield a percolation threshold at lower loadings compared with irregularly shaped particles, since the former will afford many more inter particle contacts. Here the particles are not fibrous but are spherical in nature. So no such tremendous change in conductivity is observed by adding higher volume fraction of the filler. But it was confirmed that the percolation threshold was not yet reached even for a loading of $120 \mathrm{phr}$. From the graphs it is obvious that the conductivity increases with increase of loading of the filler. We have already proved indirectly from the dielectric and magnetic studies that there is no matrix filler interaction in these composites (Anantharaman et al 1998, 1999). This in itself means that the filler goes into the matrix uniformly. If the difference in surface tension of the filler and the matrix is large then the filler will tend to aggregate and gives higher conductivity at lower loading. No such effect is observed in this case. This once again confirms that the matrix filler interaction is minimal.

\subsection{Temperature dependence}

The effect of temperature on the a.c. electrical conductivity of ceramic nickel zinc ferrite samples as well as the rubber ferrite composites were also studied in the range $303 \mathrm{~K}$ to $473 \mathrm{~K}$. It was observed that the conductivity

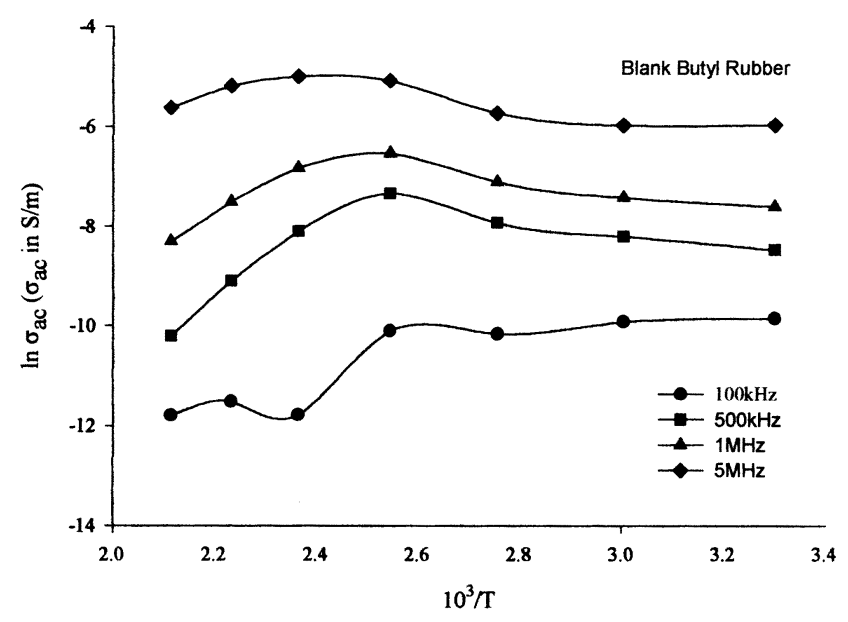

Figure 8. $\ln \sigma_{\text {a.c. }}$ vs $10^{3} / \mathrm{T}$ (blank butyl rubber). 
increases with increase of temperature for all ceramic nickel zinc samples. At low frequencies the variation is very minimal but at higher frequencies the variation is noticeable. The variation pattern of conductivity with temperature for different compositions are shown in figure 7. The influence of temperature on conductivity can be explained by considering the mobility of charge carriers responsible for hopping. As temperature increases the mobility of hopping ions also increases thereby increasing conductivity. The electrons which are involved in hopping are responsible for electronic polarization in these ferrites. The temperature dependence of conductivity for blank butyl rubber was also noted. It shows that the conductivity increases up to a temperature of $393 \mathrm{~K}$. Further increase of temperature reduces the conductivity. This decrease in conductivity at higher temperature is due to the thermal expansion of polymer. At higher temperatures the polymer density is reduced by thermal expansion and this reduces the conductivity. The change is shown graphically in figure 8 . Like butyl rubber, ferrite incorporated rubber composites also show a small reduction at higher temperature. But the conductivity values
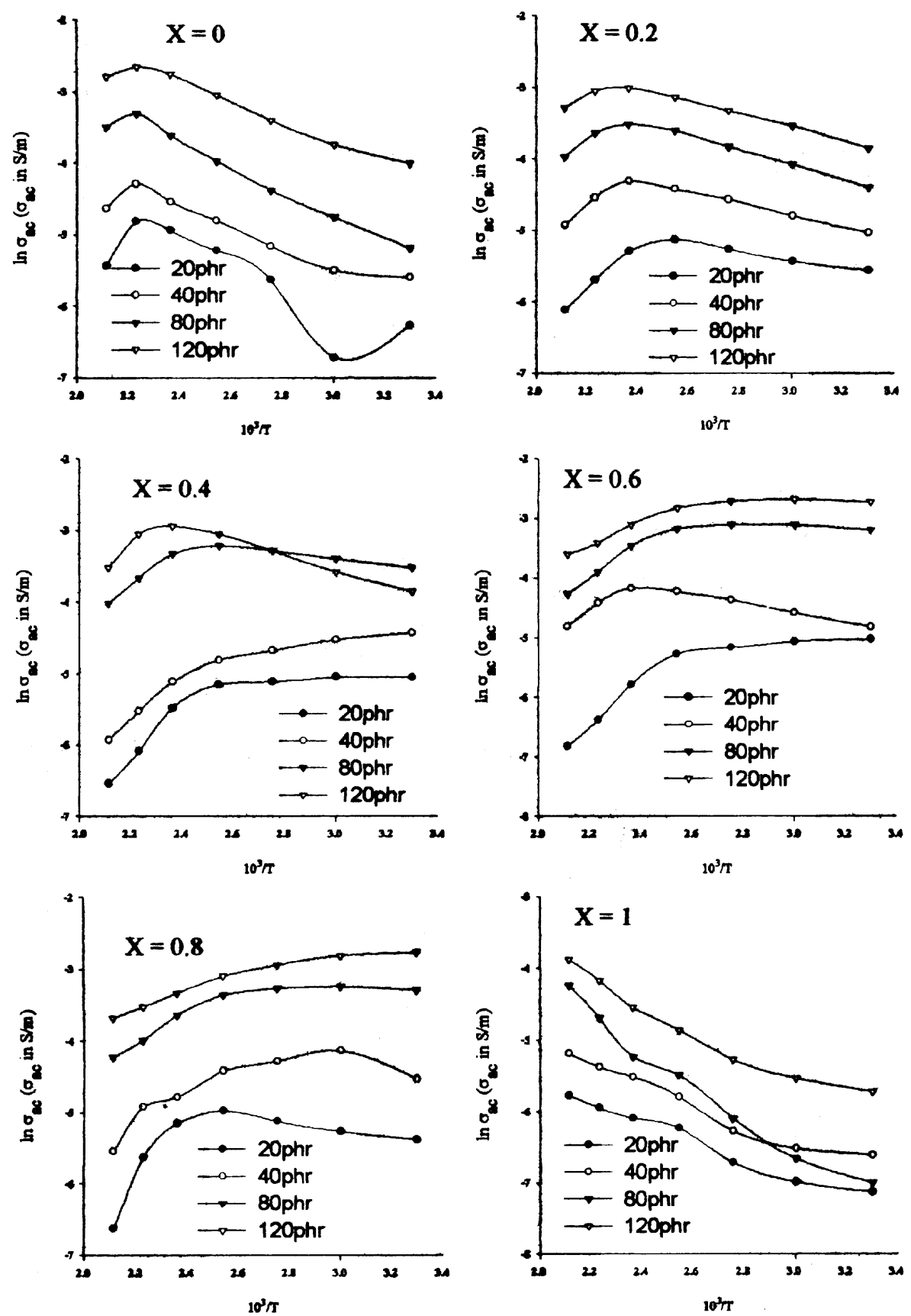

Figure 9. Temperature dependence of conductivity for RFCs (1 MHz). 
are much greater than that of blank butyl rubber. The variation pattern is depicted in figure 9 .

\section{Conclusions}

The a.c. electrical conductivity of ceramic as well as composite samples is calculated by using a simple relationship of the form $\sigma_{\text {a.c. }}=2 \pi f \tan \delta \varepsilon_{0} \varepsilon_{\mathrm{r}}$, with the data available from dielectric measurements. The results suggest that the a.c. electrical conductivity is directly proportional to the frequency. It shows an increase in conductivity with increase in frequency for both ceramic and rubber ferrite composites. The compositional dependence shows an initial increase of a.c. electrical conductivity with zinc content and reaches a maximum for $x=0.6$ thereafter it decreases. This variation can be successfully explained with the help of porosity of the ceramic samples. The variation of conductivity with volume fraction of the magnetic filler shows a continuous increase up to a loading of $120 \mathrm{phr}$. This variation remains the same for all compositions.

\section{Acknowledgements}

One of the authors (MRA) thanks the All India Council for Technical Education (AICTE), for the financial assistance received in the form of a project under TAPTEC, GOI (F.No. 8017/RDII/MAT/30/98 dated 06-03-1998). (SS) thanks DST for the financial support in the form of a fellowship. (KAM) thanks the Council of Scientific and Industrial Research, New Delhi, for the Research fellowship.

\section{References}

Abdeen A M 1998 J. Magn. Magn. Mater. 185199 Ahmed M A and Elhiti M A 1995 J. Phys. III 5775

Ahmed M A, Elhiti M A, El Nimr M K and Amer M A 1996 J. Magn. Magn. Mater. 152391

Anantharaman M R, Jagatheesan S, Sindhu S, Malini K A, Chinnasamy C N, Narayanasamy A, Kurian P and Vasudevan K 1998 Plastics, rubber and composites-Processing and applications $\mathbf{2 7} 77$
Anantharaman M R, Sindhu S, Jagatheesan S, Malini K A and Kurian P 1999 J. Phys. D: Appl. Phys. 321801

Anantharaman M R, Malini K A, Sindhu S, Mohammed E M, Date S K, Kulkarni S D, Joy P A and Philip Kurian 2001 Bull. Mater. Sci. 24623

Andrej Žnidaršiě and Miha Drofenik 1999 J. Am. Ceram. Soc. 82359

Brockman F G and White R P 1971 J. Am. Ceram. Soc. 54183

Chandra Prakash Rana and Baijal J S 1985 J. Less Common Metals 106257

Elhiti M A 1994 J. Magn. Magn. Mater. 136138

Elhiti M A 1996 J. Magn. Magn. Mater. 164187

Elhiti M A, Ahmed M A, Mosaad M M and Attia S M 1995 J. Magn. Magn. Mater. 150399

Goswami A 1996 Thin film fundamentals (New Delhi: New Age International (P) Ltd.)

Jankowski S 1988 J. Am. Ceram. Soc. 71 c-176

Kim S S, Jo S B, Gueon K I, Kim K K and Churn K S 1991 IEEE Trans. Magn. 275462

Koops C G 1951 Phys. Rev. 83121

Kulikowski J 1984 J. Magn. Magn. Mater. 4156

Mirtaheri S A, Yin J, Seki H, Mizumoto T and Naito Y 1989 Trans. IEICE E-72 1447

Pal M, Brahma P and Chakravorty D 1994 J. Phys. Soc. Jap. 63 3356

Pal M, Brahma P and Chakravorty D 1996 J. Magn. Magn. Mater. 152370

Samokhvalov A A and Rustamov A G 1964 Sov. Phys. Solid State 6749

Shaikh A M, Bellad S S and Chougule B K 1999 J. Magn. Magn. Mater. 195384

Stoyan I Ganchev, Jaybhattacharyya, Sasan Bakhtiari, Nasser Qaddoumi, Deborah Brandenburg and Reza Zoughi 1994 IEEE Trans. Microwave Theory \& Technol. 4218

Smit J and Wijn H P J 1959 Ferrites (Eindhoven: Philips Technical Library)

Sung-Soo Kim, Dae-Hee Han and Sung-Baeg Cho 1994 IEEE Trans. Magn. 304554

Terje A Skotheim 1986 Handbook of conducting polymers (New York, USA: Marcel Dekker Inc.) Vols I and II

Yootarou Yamazaki and Minuru Satou 1973 Jap. J. Appl. Phys. 12998

Yoshiyuki Naito, Jifang Yin and Tetsuya Mizumoto 1998 Electron. \& Commun. Jap. $\mathbf{7 1} 77$ 